\title{
Electronic and Transport Properties of Bilayer Phosphorene Nanojunction: Effect of Paired Substitution Doping
}

\author{
Vivekanand Shukla,* Rameshwar L. Kumawat, Naresh K. Jena, Biswarup Pathak, and Rajeev Ahuja
}

Cite This: ACS Appl. Electron. Mater. 2021, 3, 733-742

Read Online

ABSTRACT: Electron transport in bilayer phosphorene is studied using the first-principles and nonequilibrium Green's function formalism. We have explored the interlayer properties of a vertically stacked bilayer structure with paired substitutional doping. The electron transport properties are calculated in bilayer phosphorene and compared with substitutional doping, which shows the tunable anisotropic nature of doped phosphorene in the $I-V$ characteristics. Further, to understand the role played by dopants, the quantum transport properties of monolayer-bilayer monolayer (ML-BL-ML) nanojunction are studied with and without dopants. The interlayer direction-dependent current

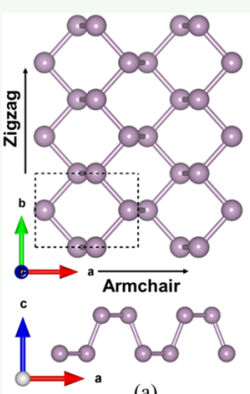

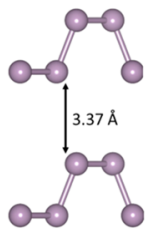

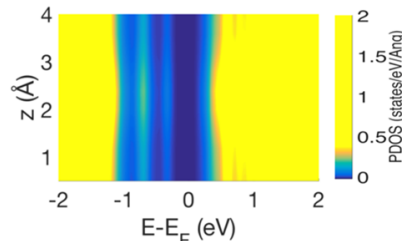

(c)

characteristics are discussed in different setups. This suggests that the dopants play a crucial role in the interlayer current and further provided rectifying behavior in the zigzag direction. Fano resonance is also observed as an effect that arises from the hydrogen-terminated edges interacting with the second layer. Our study demonstrates significant tuning of the electronic transport properties of the bilayer phosphorene implying its potential application in electronic devices.

KEYWORDS: phosphorene, 2D materials, bilayer, density functional theory, nonequilibrium Green's function, quantum transport, $I-V$ characteristics, Fano resonance, rectification

\section{INTRODUCTION}

In the last two decades, two-dimensional (2D) materials have attracted us due to their wide variations in structural, chemical, physical, and electronic properties, not to mention about their novel applications in sensing, energy storage, photocatalysis, and so on. ${ }^{1-6}$ Two-dimensional materials can be used in nanoscale electronic devices as electrodes, dielectrics, or semiconductors. ${ }^{1-4,7-10}$ The key advantage of $2 \mathrm{D}$ materials is the property that makes them naturally suited for a type of integration, which is not possible with any three-dimensional (3D) material, that is, forming heterostructures by stacking $2 \mathrm{D}$ materials together. Recently, significant effort has been made to making vertical and lateral heterojunctions of these 2D materials $\mathbf{s}^{1,11,12}$ that can behave like a fundamental component of the electronic devices like photodiode, field-effect transistor (FET), rectifier, etc. ${ }^{13-17}$ Several vertical and lateral nanojunctions have already been reported both theoretically and experimentally, such as graphene, ${ }^{7}$ hexagonal boron nitride (h$\mathrm{BN}){ }^{8} \mathrm{MoS}_{2}$, and $\mathrm{WS}_{2}{ }^{9,109,10}$ In addition to all of these, recently, a monolayer of black phosphorus (BP) known as phosphorene has been exfoliated from bulk, and it has shown promising applications in nanoscale electronic devices such as sensing, ${ }^{18-21}$ energy storage, ${ }^{22}$ and photocatalysis. ${ }^{23}$ It is noted that $\mathrm{BP}$ has a direct band gap of $0.3 \mathrm{eV}$ and mobility of $\sim 10000 \mathrm{~cm}^{2} / \mathrm{VS}$, whereas monolayer phosphorene also has a direct band gap of $0.9 \mathrm{eV}$ calculated with generalized gradient approximation (GGA) functional. ${ }^{18-21,24-27}$ BP has a highly anisotropic electronic structure in the $x-y$ plane that is dependent on the number of layers. A few layers of phosphorene are more interesting than their monolayer. A few layers of phosphorene also have highly anisotropic electron transport properties, which is supported by experimental work as well as theoretical calculation of the effective masses of holes and electrons. ${ }^{28-30}$ Several theoretical and experimental studies have been conducted to tune the anisotropic electronic and transport properties of phosphorene from both the electronic structure and device perspective. ${ }^{18,28-31}$ Apart from doping, the anisotropic property of BP can also be tuned with the applied strain and electric field. ${ }^{31-35}$ Highly tunable electronic and transport property as well as high on/off ratio of BP make it suitable for applications in nanoelectronics devices. $^{24-26}$

Therefore, significant effort has been made to study the electronic and transport properties of BP with layer dependence that has been reported elsewhere. ${ }^{27,31,33,34,36,37}$ There are

Received: October 8, 2020

Accepted: February 1, 2021

Published: February 11, 2021

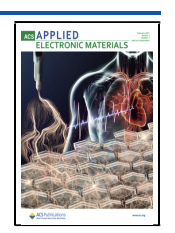


also several reports showing the band gap tuning of this phosphorene with nanostructure width (nanoribbon). ${ }^{38,39}$ Also, bilayer phosphorene (Bi-P) flakes have been extensively studied both experimentally and theoretically for electronic and transport properties. ${ }^{40,41}$ These bilayer flakes show exciting features along with bilayer nanoribbon. The energy gap of the bilayer with respect to its width and length and the corresponding atomic termination have also been reported. $^{27,40,41}$

Chemical doping has been a very convenient way to tune the carrier concentration of a semiconductor. Monolayer phosphorene shows drastically changed electronic properties with doping like metal element and a single atom from groups I, III, $\mathrm{V}$, and VI. ${ }^{39,41-46}$ Another report shows that multiple boron (B) and nitrogen (N) doping prefers a highly ordered structure. $^{45,47}$ The higher concentration of arsenic atoms is also studied experimentally and theoretically. ${ }^{48-52}$ StoneWales (SW) deformations are also discussed before. ${ }^{53}$ Our current electronic world is mostly based on silicon ( $\mathrm{Si}$ ); therefore, Si doping can be the right choice of study and can be of particular importance, and the atomic radius of $\mathrm{Si}$ is also similar to that of phosphorus (P). ${ }^{41}$

Motivated from the high mobility and peculiar anisotropic properties of phosphorene and previously attempted various doping effects, in this report, we concentrate on the electronic and quantum transport properties of free-standing bilayer phosphorene flakes along the armchair and zigzag directions. We assume that the best way to get a phosphorene bilayer flake is through overlapping of a phosphorene bilayer. We further introduced the paired doping effect in this $\mathrm{Bi}-\mathrm{P}$ flake to understand the impact of substituents on the electronic and transport properties in the higher-bias regime. Our results reveal significant tuning of the Bi-P anisotropy owing to paired doping, implying its potential application in electronic devices. We also introduced a nanojunction made of two vertically stacked phosphorene doped with $\mathrm{Si}$ and $\mathrm{S}$ atoms and tried to calculate the electronic and transport properties. In contrast, nanojunctions show that current reductions relative to bilayer are lower than that of graphene cases due to the strong interlayer coupling in Bi-P. ${ }^{54}$ Finally, we discussed the interesting rectifying behavior observed in the doped zigzag direction, which was eventually missing in the armchair nanojunction.

\section{COMPUTATIONAL DETAILS}

The first-principles-based density functional theory (DFT) framework is used for this work. ${ }^{55,56}$ We first relaxed the monolayer and bilayer unit cells in the Vienna $\mathrm{Ab}$ initio Simulation Package (VASP). ${ }^{57}$ Projected augmented wave (PAW) was taken into account to define the ion-electron interaction, and exchange-correlation has been described by GGA in the Perdew-Burke-Ernzerhof (PBE) scheme. ${ }^{58-60} \mathrm{~A}$ plane-wave cutoff of $500 \mathrm{eV}$ is used for kinetic energy. All of the atomic forces were relaxed up to energy less than 0.01 $\mathrm{meV}$, and the corresponding forces on each atom up to energy less than $0.01 \mathrm{eV} / \AA$. We used the van der Waals (vdW) interaction during our calculations as implemented in VASP for D3 Grimme correction. ${ }^{61}$ After defining the unit cell, we used the SIESTA code ${ }^{62,63}$ to relax the supercell, and GGAPBE functional has been used in the SIESTA calculation. ${ }^{58}$ The atomic core electrons were modeled with TroullierMartins norm-conserving pseudopotentials. ${ }^{64}$ A mesh cutoff of $200 \mathrm{Ry}$ was used for real space integration with double- $\zeta$ polarized (DZP) basis set, ${ }^{65}$ and Brillouin zone sampling, with a mesh of $1 \times 2 \times 2$, has been used in the supercell. It is worth mentioning that DZP gives good results, although to get the same result as plane-wave code, one must use the complete basis sets. All of these structures were fully relaxed with the conjugate gradient (CG) algorithm considering the fact that residual forces in each component of the atoms were smaller than $0.01 \mathrm{eV} / \AA ̊$. After relaxing the supercell, we designed the transport setup, and the setup was also relaxed with the same accuracy of $1 \times 4 \times 1 k$-point grid. After this, the transport calculations were performed using the quantum transport code TranSIESTA implemented along with SIESTA. ${ }^{66}$ This method combines DFT and the nonequilibrium Green's function (NEGF) formalism. The basis sets employed in the transport calculation were the same as those used during the structural optimization processes. When a given voltage is applied, the current is allowed to flow across the system. The electric current $(I)$ is further calculated using the Landauer approach, and this can be obtained from the integration of the transmission curve as ${ }^{67}$

$$
I\left(V_{\mathrm{b}}\right)=\frac{2 e}{h} \int_{\mu_{\mathrm{R}}}^{\mu_{\mathrm{L}}} T\left(E, V_{\mathrm{b}}\right)\left[f\left(E-\mu_{\mathrm{L}}\right)-f\left(E-\mu_{\mathrm{R}}\right)\right] \mathrm{d} E
$$

where $I\left(V_{\mathrm{b}}\right)$ represents the electric current under applied bias voltages, $e$ is the electron charge, $h$ is Planck's constant, $f(E-$ $\left.\mu_{\mathrm{L} / \mathrm{R}}\right)$ is the Fermi-Dirac distribution function of the $\mathrm{L}$ and $\mathrm{R}$ electrodes, $\mu_{\mathrm{L} / \mathrm{R}}\left(\mu_{\mathrm{L} / \mathrm{R}}=E_{\mathrm{F}} \pm \frac{V_{\mathrm{b}}}{2}\right)$ is the chemical potential, which can move up and down according to the Fermi energy $E_{\mathrm{F}}$, and $T\left(E, V_{\mathrm{b}}\right)$ is the quantum transmission probability of the electrons, which can be given as follows

$$
T\left(E, V_{\mathrm{b}}\right)=\Gamma_{\mathrm{L}}\left(E, V_{\mathrm{b}}\right) G\left(E, V_{\mathrm{b}}\right) \Gamma_{\mathrm{R}}\left(E, V_{\mathrm{b}}\right) G^{\dagger}\left(E, V_{\mathrm{b}}\right)
$$

where the coupling matrices are given as $\Gamma_{\mathrm{L} / \mathrm{R}}=i\left[\sum_{\mathrm{L} / \mathrm{R}}-\right.$ $\left.\sum_{\mathrm{L} / \mathrm{R}}^{\dagger}\right]$ and the NEGFs for the scattering region given as $G\left(E, V_{\mathrm{b}}\right)=\left[E \times S_{\mathrm{s}}-H_{s}[\rho]-\sum_{\mathrm{L}}\left(E, V_{\mathrm{b}}\right)-\sum_{\mathrm{R}}\left(E, V_{\mathrm{b}}\right)\right]^{-1}$, where $S_{\mathrm{s}}$ is the overlap matrix, $H_{\mathrm{s}}$ is the Hamiltonian matrix, $\sum_{\mathrm{L} / \mathrm{R}}=V_{\mathrm{s}_{\mathrm{L} / \mathrm{R}}} g_{\mathrm{L} / \mathrm{R}} V_{\mathrm{L} / \mathrm{R}} s$ is the self-interaction energy, $\sum_{\mathrm{L} / \mathrm{R}}$ is a molecule electrode that takes into account $\mathrm{L} / \mathrm{R}$ electrodes in the central scattering region, $g_{\mathrm{L} / \mathrm{R}}$ is the surface $\mathrm{L} / \mathrm{R}$ Green's function, and $V_{\mathrm{L} / \mathrm{R}} s=V_{\mathrm{S}_{\mathrm{L} / \mathrm{R}}}^{\dagger}$ is the coupling matrix between $\mathrm{L} / \mathrm{R}$ electrodes and the scattering region. ${ }^{19,21,27,67}$

\section{RESULTS AND DISCUSSION}

3.1. Structural and Electronic Properties. Monolayer phosphorene has a rectangular cell with lattice parameters $a=$ 4.60 and $b=3.29 \AA$ with four atoms in the unit cell. ${ }^{27,32}$ It shows a direct band gap with a calculated gap of $0.9 \mathrm{eV}$ in our GGA-PBE calculation well relevant to the previous reports. ${ }^{18,27,32,52}$ It is well known that the GGA underestimates the band gap of phosphorene, and it can further be tuned by HSE06 functional and quasi-particle calculations such as GW, which matches well with the experimentally measured band gap of $1.7 \mathrm{eV}$. We would further go ahead with GGA-PBE because the number of atoms in the cell makes it computationally expensive, and apart from the band gap, dispersion of the bands does not change with HSE06. Similar to other 2D materials, there are several stackings possible for bilayer phosphorene, such as $\mathrm{AA}, \mathrm{AB}, \mathrm{A}-\mathrm{C}(\mathrm{I})$, and $\mathrm{AC}(\mathrm{II})$. All of these stacked bilayers show the direct band gap property in the electronic structure; these stackings have been discussed in 
several reports. ${ }^{68,69}$ Apart from this, $\mathrm{A} \delta$ stacked bilayer is also possible as a stacking fault and gives indirect band gap characteristics reported previously. ${ }^{69}$ Although we have also explored all of the stacking, at the moment, for this work, we would focus on AA stacked bilayer BP, which is slightly higher in energy than that of $A B$ stacking. Figure la shows the side

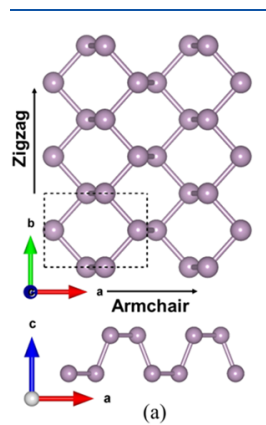

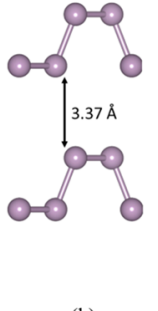

(b)

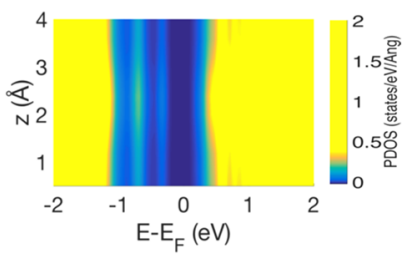

(c)
Figure 1. (a) Armchair and zigzag view of the phosphorene unit cell, (b) schematic picture of AA stacking, and (c) the density of state for bilayer phosphorene.

and top views of AA stacked bilayer phosphorene with all of the atoms on the top of each other to the respective atoms. The calculated unit cell lattice parameter is $4.55 \times 3.30 \AA^{2}(a \times$ $b$ ), and interlayer spacing for this bilayer cell is $3.37 \AA$ (Figure 1b). Further, Figure 1c shows the contour plot for the electronic density of states (DOS) of the AA stacked bilayer, which clearly indicates that the bilayer phosphorene has a lower band gap than that of the monolayer and the estimated gap is $\sim 0.36 \mathrm{eV}$.

Unlike graphene, bilayer phosphorene shows a considerable variation in the band gap depending upon the number of layers. This reduction in the band gap originates due to extra lone pair electrons present in phosphorus (P) atoms, which is entitled to the hybridization in between the layers. This hybridization becomes weaker when the bilayer is twisted by $90^{\circ}$, which has already been discussed in our earlier report. ${ }^{27}$

We fixed the interlayer spacing at $3.37 \AA$, which we obtained from the VASP calculation with dispersion correction using the DFT-D3 method. SIESTA code is used for further calculation of supercells, where we took $4 \times 3$ supercell with 48 atoms per layer with $\mathrm{Si}-\mathrm{S}$ substitutional pair. Among the several possible paired substitutions, the first one is the $\mathrm{Si}$ in the lower sublayer of top phosphorene layer and $S$ doping in the upper sublayer of the bottom phosphorene layer $\left(\mathrm{Si}_{\mathrm{L}} \mathrm{S}_{\mathrm{U}}\right)$. The second configuration is the Si doping in the upper sublayer of the top layer and $S$ doping in the lower sublayer of the bottom phosphorene layer $\left(\mathrm{Si}_{\mathrm{U}} \mathrm{S}_{\mathrm{L}}\right)$. The third one is the $\mathrm{Si}$ doping in the lower sublayer of the top layer and $S$ doping in the lower sublayer of the bottom layer $\left(\mathrm{Si}_{\mathrm{L}} \mathrm{S}_{\mathrm{L}}\right)$. We tried all of these different configurations of substitution, both for the monolayer and bilayer phosphorenes. The calculated formation energies, respective doped structures, and discussions can be found in the Supporting Information (Figure S1). The formation energies suggested that the $\mathrm{Si}$ substitution in the lower sublayer of the first layer and the $S$ substitution in the upper sublayer of the second layer $\left(\mathrm{Si}_{\mathrm{L}} \mathrm{S}_{\mathrm{U}}\right)$ is exothermic, and it can be experimentaly realized as shown in Figure 2a. This configuration of doping will be taken for our subsequent analysis. The observed $\mathrm{Si}-\mathrm{P}$ bond lengths are 2.32 and $2.35 \AA$, and the $\mathrm{S}-\mathrm{P}$ bond lengths are 2.24 and $2.58 \AA$. It has already been reported that $\mathrm{Si}-\mathrm{Si}$ substitution in bilayer phosphorene is

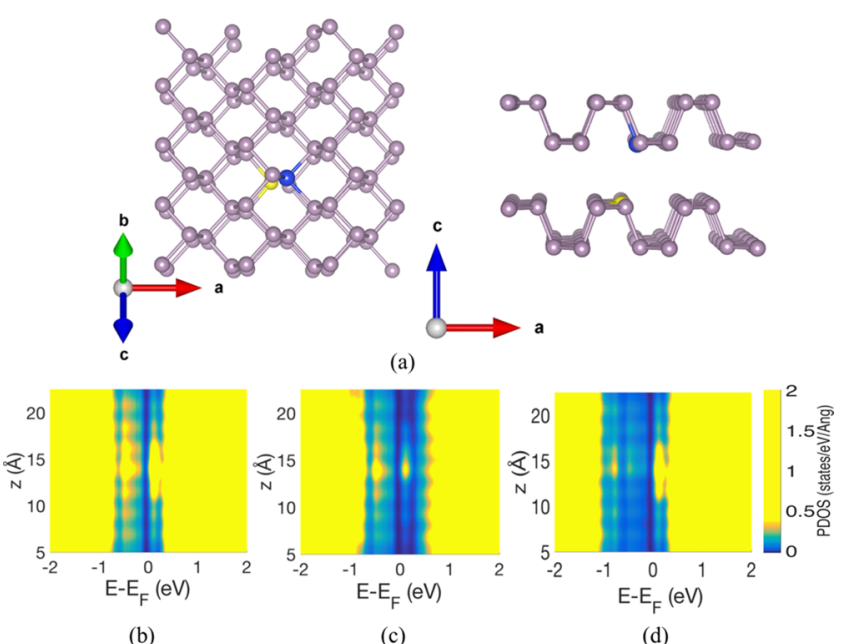

(b)

(c)

(d)

Figure 2. (a) Top and side views of Si-S-substituted bilayer phosphorene system. (b) Total electronic density of states for bilayerdoped phosphorene system and projected density of states for (c) Sidoped layer and (d) S-doped layer. The blue color indicates the gap, and the gradient toward the yellow colors shows the states; the contour is plotted in the $z$-direction as an axis to understand the doping position in the plot. Color code: blue $(\mathrm{Si})$, violet $(\mathrm{P})$, yellow (S).

suitable on top of each other in the A-A stacking configuration. ${ }^{41}$ Once we have a relaxed structure with fixed cell size, we further used a sufficiently large $8 \times 8 \times 1 k$-point grid for electronic structure calculations. Figure $2 \mathrm{~b}$ shows the electronic density of states (DOS) for the bilayer-doped system. There are states available close to the Fermi level, both upper and lower sides of the Fermi value. These states originated from the $\mathrm{Si}$ and $\mathrm{S}$ dopants, which overcome the band gap found in the pristine bilayer. Further, to understand the influence of each dopant on electronic structure, density is projected on the top and bottom layers shown in Figure $2 \mathrm{c}$ for the Si-doped layer and in Figure $2 \mathrm{~d}$ for the S-doped layer. It is evident that the state below the Fermi level arises predominantly due to $\mathrm{Si}$ doping in the bottom panel, and above the Fermi energy, the states belong to the $S$ doping in the upper layer. In our previous report on doped monolayer, we have already concluded that the charge in the S-doped layer was more concentrated on the dopant $S$ atom, whereas the charge in the Si-doped layer was more diffused around the closed P atoms. ${ }^{18}$ Overall, from the electronic DOS picture, it is evident that the doping gives us the state that resembles $\mathrm{p}$ type doping in the upper layer. On the other hand, lower layer doping provides us with the state also above the Fermi level as well as below the Fermi level. Previous reports suggested that the monolayer with $\mathrm{Si}$ and $\mathrm{S}$ doping exhibits a metallic band around the Fermi due to the strong hybridization of $s p$ orbitals in dopant and phosphorus. Here, because of the bilayer and paired doping, we have states along with the Fermi, but we also have a small band gap in bilayer electronic DOS. S dopant has a strong state in the conduction band region, while Si dopant has a dominant state in the valence band. These two dopants suppress the overall band gap.

3.2. Electronic Transport Properties. The isoelectric bilayer doping discussed in the last section motivated us to study the electronic transport properties of bilayer phosphorene along with the doping. These calculations are performed in the TranSIESTA code implemented with nonequilibrium 


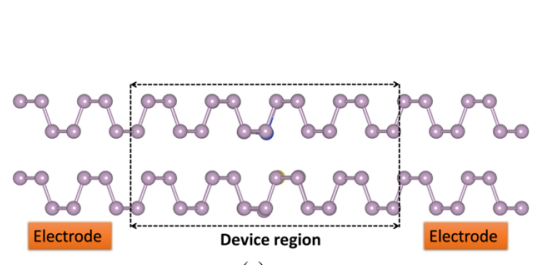

(a)

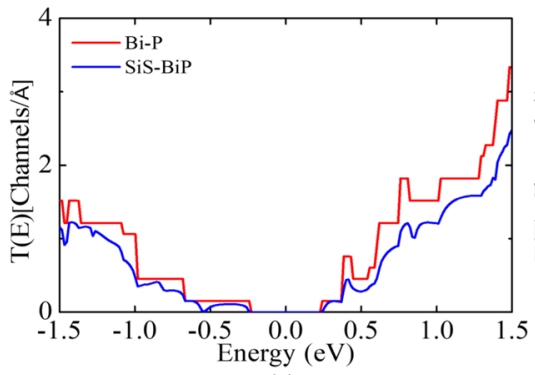

(c)

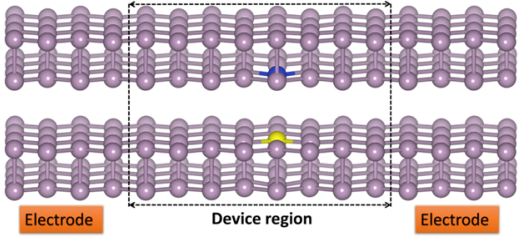

(b)

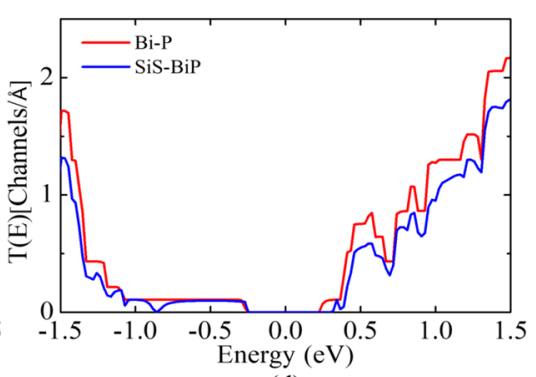

(d)

Figure 3. Schematic of electron transport devices on bilayer and doped phosphorene systems. Central device regions are connected with semiinfinite electrodes in (a) armchair and (b) zigzag directions. Zero-bias transmission for doped and pristine bilayer devices in (c) armchair and (d) zigzag directions.

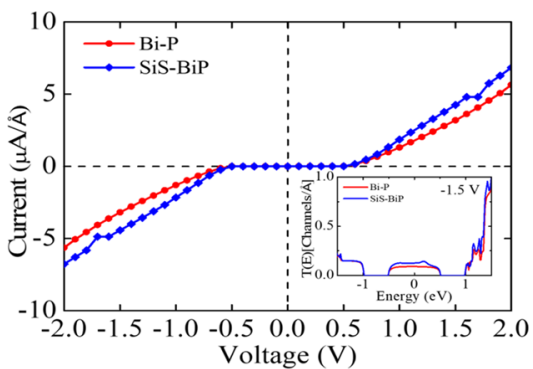

(a)

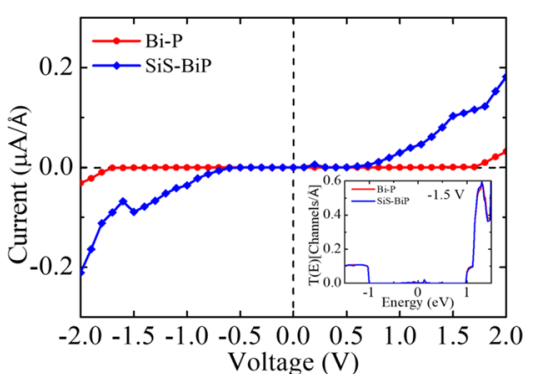

(b)

Figure 4. $I-V$ characteristics for the pristine and $\mathrm{Si}-\mathrm{S}$-substituted bilayer phosphorene system in (a) armchair and (b) zigzag directions. The insets show the elevated bias transmissions at the bias of $-1.5 \mathrm{~V}$.

Green's function formalism in conjugation with DFT. To perform the transport calculations, the system is divided into three sections: two semi-infinite electrodes extended infinitely, and a central region where scattering processes take place. These electrodes have been modeled for the perfect bulk properties of bilayer phosphorene. The central region, as can be seen in Figure 3, is connected with two electrodes in transport calculations. The schematic nanoscale device can be seen in Figure 3a,b, for both armchair and zigzag directions. First, the transport properties in pristine bilayer are in good agreement with various theoretical studies and show perfect anisotropic behavior. This anisotropic behavior of phosphorene is intrinsic and results from the asymmetric band dispersion between G- $X$ and G- $Y$ directions, which results in highly anisotropic effective masses of electrons and holes and eventually carrier mobilities in the respective directions. The $\mathrm{G}-X$ band disperses rather strongly and corresponds to lower effective mass in compression to the G- $Y$ band, which has lower dispersion. ${ }^{27}$ As we are interested in isoelectric doping to understand the behavior of dopant in electronic transport, we further extended our study to the doped system. We maintained the width of the $2 \mathrm{D}$ structure with a previously discussed supercell in the DOS study. Thus, our doping makes a linear chain of dopant in the orthogonal direction of the transport, unlike the nanoribbon, and in the transport direction, it is terminated with perfect bilayer phosphorene.
The presence of a semi-infinite electrode modifies the electronic property of $\mathrm{Si}-\mathrm{S}$-substituted bilayer system because the Fermi level is now fixed to the semi-infinite lead of bilayer phosphorene. The zero-bias transmission for the pristine bilayer and $\mathrm{Si}-\mathrm{S}$-substituted bilayer are shown in Figure 3c,d. There is a transport gap of around $0.36 \mathrm{eV}$ in both the zigzag and armchair directions, which results from our semiconductor electrode (phosphorene). Doped systems in both the devices also show the same transmission gap because the electrons would be injected through the pristine bilayer phosphorene in the same manner as the bilayer device. Further, we can clearly see that the bilayer has a steplike transmission curve, which results from the $\gamma$ point calculation. We also tried the same with higher $k$-mesh, but the qualitative picture does not change much, and most of the previous studies have $\gamma$ point transport calculations for such types of devices. The overall transmission decreases for the Si-S-substituted bilayer due to the $\mathrm{Si}$ and $\mathrm{S}$ scattering centers. In the zero-bias regime, these scattering centers block the transmission channels and decrease the transmissions. Further, if the doping effect has to be understood, we need to study the system under a higher-bias regime when it overcomes the band gap of the bilayer electrode. The electron bias was increased step by step with increments of $0.1 \mathrm{~V}$ to achieve the full convergence of nonequilibrium density, and further, the transmission was calculated. 


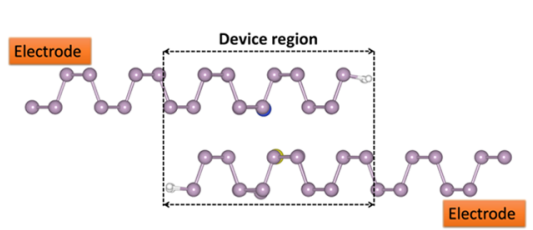

(a)

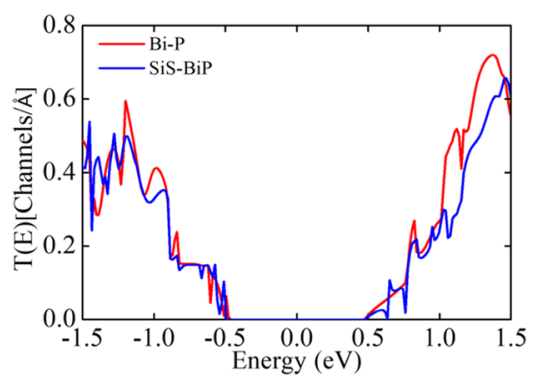

(c)

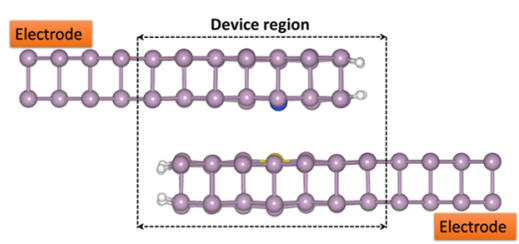

(b)

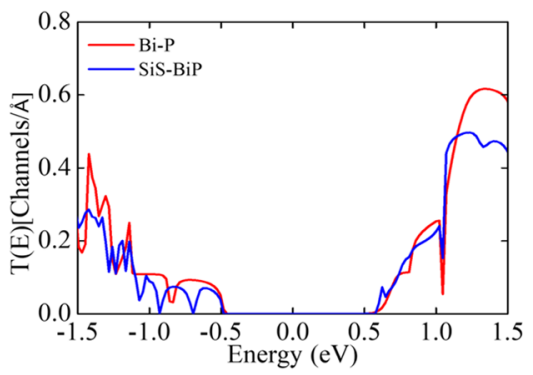

(d)

Figure 5. Schematic illustration of H-terminated ML-BL-ML phosphorene nanojunction device setup in (a) armchair and (b) zigzag directions. Zero-bias transmission for both (c) armchair and (d) zigzag pristine and doped nanojunctions.

The current-voltage $(I-V)$ characteristics were estimated using the Landauer-Büttiker formula at every bias transmission at the interval of $0.1 \mathrm{~V}$. Figure $4 \mathrm{a}, \mathrm{b}$ shows the $I-V$ characteristics for both the bilayer pristine and periodically $\mathrm{Si}-$ S-doped system where currents are higher in the doped device than the pristine one in both the armchair and zigzag cases. It has already been reported that doping can enhance the conductivity of phosphorene monolayer, but we get the same trend in an isoelectrically doped system. There is no clear evidence of asymmetric electron variation in the upper or lower layer, and it happens because of the symmetric contacts and electron transmission path. To understand the higher current in the doped system compared to the pristine one, the elevated bias transmissions (at $-1.5 \mathrm{~V}$ ) are shown in the insets of Figure 4a,b. From the transmission at an elevated bias of $-1.5 \mathrm{~V}$, it is evident that when the bias is increased, the scattering center starts taking part in electron transport and we have observed enhanced transmission because of $\mathrm{Si}$ and $\mathrm{S}$ dopants.

In the armchair case, the doped system shows higher transmission than the pristine case. This higher transmission in a doped system results in a higher current. On the other hand, the zigzag direction shows negligible transmission around the Fermi level for pristine cases; hence, the current is low compared to the armchair directions. Moreover, we have smaller current values in Figure $4 \mathrm{~b}$ for both the doped and pristine devices than the corresponding armchair directions because of the highly anisotropic transport property in phosphorene, and the detailed discussion about the transport anisotropy can be found elsewhere. ${ }^{27}$ Overall, the current variations with and without doping are higher in the zigzag direction than in the armchair direction, as evident from Figure $4 a, b$. This suggests that the doping effect in the zigzag direction is more prominent than in the armchair direction, which further motivated us for the statement that the anisotropic properties in $I-V$ characteristics get tuned with the number of the layer and substitutional doping in phosphorene can further tune the anisotropy in the system.

After having a detailed view of the electronic and transport properties of the pristine and doped two different bilayers in the armchair and zigzag directions, we now introduce the homogeneous and doped nanojunction. Two layers of phosphorene in the form of AA stacking were put on the top of each other, and one of the directions was terminated with hydrogen for passivation. Here, the two layers of $\mathrm{BP}$ in the form of AA stacking were put on each other, and then in either direction, dangling bonds were passivated by hydrogen atoms to prevent the edge reconstructions. This passivation gives stability to the device during the structural relaxation. It gives the monolayer-bilayer-monolayer ( $\mathrm{ML}-\mathrm{BL}-\mathrm{ML}$ ) device architecture, where the central region has a bilayer of the armchair configuration, as in Figure 5a, and the zigzag configuration, as in Figure $5 \mathrm{~b}$. The monolayers in this ML$\mathrm{BL}-\mathrm{ML}$ nanojunctions are designed to have an armchair and zigzag form. These nanojunctions are created deliberately to understand the direction dependence of phosphorene and further the isoelectric doping effect. Further, the transport properties would be estimated from nearly the same length of the scattering region in these two devices. This also provides us the opportunity to understand the role played by the dopants on the interlayer current because we create asymmetric devices by terminating either direction with hydrogen, and the scattering region is doped by donor and acceptor atoms. In these devices, a potential well is formed in this scattering region (bilayer region) shown in zigzag and armchair directions in Figure 5a,b, respectively. Hence, the device region is constituted by partially overlapped doped phosphorene flakes. In the present setup, electron current should flow from one phosphorene layer to others. It breaks the left-right symmetry and may behave like a $\mathrm{p}-\mathrm{n}$ nanojunction. Unlike the bilayer phosphorene, the electrodes in this setup are in the form of monolayer, which is also against the periodicity of the device. On the left-hand side, the electrode belongs to the upper layer geometry, and on the right-hand side, the electrode is attached to the lower layer, which gives different geometries for the lead in the vacuum direction. To encounter this issue, buffer layers were added to extend the lead, which also gives the bulk electronic structure for the lead with no extra computing cost in TranSIESTA. This is necessary to prevent the charging effect in the device.

Computed zero-bias transmissions for nanojunctions have a more significant transmission gap in both the armchair and 
zigzag directions than the bilayer system owing to the electronic structure of the electrode, which now has monolayer geometry. The overall zero-bias transmission is the same as the bilayer system in Figure 5c,d; doping suppresses the transmission function due to scattering effects. Zero-bias transmission and its direction dependence have already been explained in our previous report. ${ }^{27}$ It is essential to study this system in a higher-bias regime to understand the asymmetric and doping effects; the bias increased again in a periodic manner with $0.1 \mathrm{~V}$ per step.

Figure 6a shows the $I-V$ characteristics for the armchair direction, and electron flow in positive bias shows that the

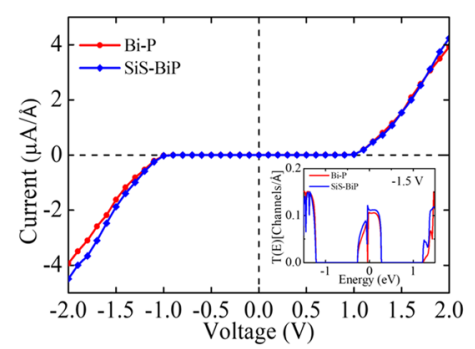

(a)

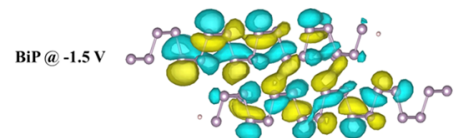

(b)

$1^{\text {st }}$ channel

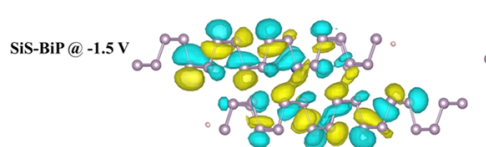

(d)

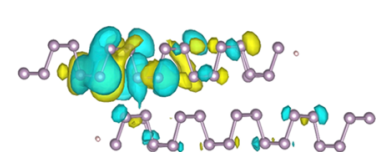

(c)

$2^{\text {nd }}$ channel
Figure 6. (a) $I-V$ characteristics of $M L-B L-M L$ nanojunction devices in the armchair direction (inset shows the elevated bias transmission at $-1.5 \mathrm{~V}$ for both doped and pristine devices). ( $\mathrm{b}-\mathrm{e}$ ) First and second eigenchannels at $-1.5 \mathrm{~V}$ for pristine and $\mathrm{Si}-\mathrm{S}$ substituted armchair nanojunction devices.

electron has to pass from the Si-doped layer to the S-doped layer. The first thing we observed in the armchair nanojunction was that the current variation in doped and pristine systems is lower than that of the bilayer device. The second, the overall current is lower than that of bilayer systems due to the lower electron density in the monolayer electrode for nanojunction. There is also a tunneling barrier for this nanojunction. This reduction is lower than it occurs in graphene nanojunction ${ }^{54}$ because bilayer phosphorene has a higher interlayer coupling than bilayer graphene due to extra lone pair electrons in phosphorus atoms, as discussed in the previous section. Further, $I-V$ characteristics for doped and pristine nanojunction in the armchair direction give quite the same current in the positive-bias direction. Still, in a negative-bias direction, the doped system has a slightly higher current than the pristine one. To understand this small rectification, we have plotted the biased transmission at $-1.5 \mathrm{~V}$ in the inset of Figure 6a. The Fano resonance can clearly be seen in the transmission for both the nanojunctions, which arises from the tunneling through the edges of the phosphorene nanojunction. This happens because of reconstruction of the edge in armchair device where hydrogens at the edges moved toward the counter layer of phosphorene. This allows interaction between the hydrogens and phosphorous from the other layer. The overall transmission is slightly higher for the doped system than the pristine one, which eventually gives rise to higher current.

Further, to get insight into this variation in transmission function, we also plotted the eigenchannels for this transmission behavior at a $-1.5 \mathrm{~V}$ bias, which was calculated in the Inelastica package. ${ }^{70}$ Figure $6 \mathrm{~b}-\mathrm{e}$ shows that the first (1st) and most dominating eigenchannels are the same for the doped and pristine systems, but the second (2nd) eigenchannels have an intersection between these layers exactly at the dopant site. This extra interaction is only seen in the doped system at the negative bias, where electrons are injected from the lower layer to the upper layer. It occurs because charges are more delocalized in the Si-doped layer; in contrast, in S-doped layer, charge density is more localized in the dopant position, which gives rise to these extra channels in the negative-bias regime. Second, eigenchannels are the less dominant ones, which is the reason behind the negligible rectification in this nanodevice.

After this less rectifying armchair nanojunction, let us move to the zigzag nanojunction, where it has already been discussed that lower current is expected due to the directional dependence of BP. In the zigzag direction, however, the overall transmission curve is the same as the bilayer electrode system but with a lower magnitude (Figure 5d). Exciting results are observed in the zigzag direction $I-V$ curve, where doped device possesses a higher current in positive bias than in negative bias, as shown in Figure 7a. This is because when an electron is getting transported from the Si-doped layer to the S-doped layer, the current is higher than that of the other direction. However, the overall current is less than the bilayer system in the zigzag direction as well as nanojunction in the armchair direction, which preserves the anisotropic nature of BP. To discern this rectification ratio, we should look at the

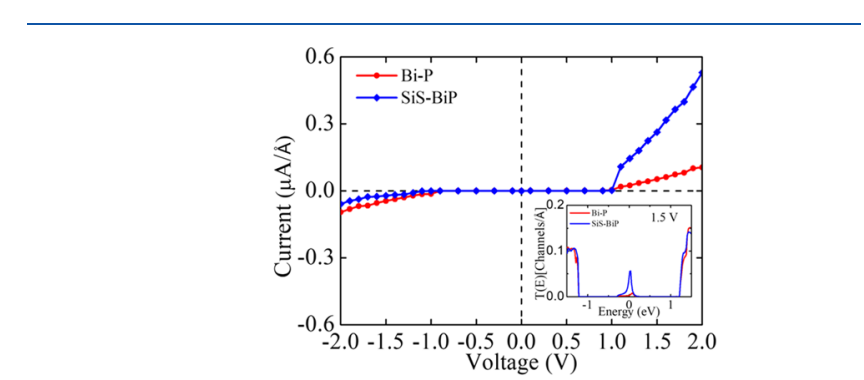

(a)

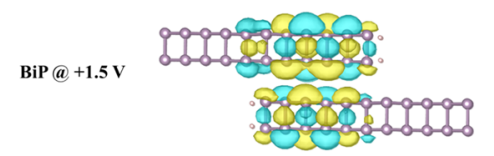

(b)

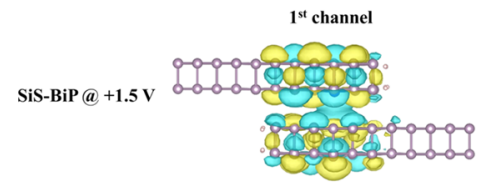

(d)

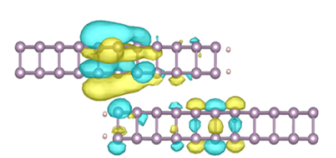

(c)

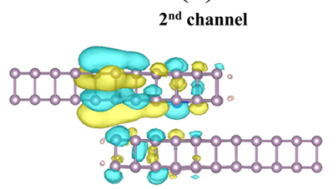

(e)
Figure 7. (a) $I-V$ characteristics for pristine and $\mathrm{Si}-\mathrm{S}$-substituted ML-BL-ML zigzag nanojunction phosphorene system (the inset shows the elevated bias transmission at $1.5 \mathrm{~V}$ for both doped and pristine devices). ( $\mathrm{b}-\mathrm{e}$ ) First and second eigenchannels at $1.5 \mathrm{~V}$ for pristine and $\mathrm{Si}-\mathrm{S}$ zigzag nanojunction devices. 
transmission function at a +1.5 bias regime for both the doped and pristine systems, which is presented in the inset of Figure 7a. It is quite clear that overall transmissions are lower than that of armchair nanojunction, but we can see that the doped nanodevice has a higher transmission than the pristine one. This means that dopants play a dominant role in electron transport at a relatively higher bias in zigzag nanojunction, which was also seen in the doped bilayer case. For an in-depth understanding, we further plotted the eigenchannels for these two nanodevices at the Fermi level for $+1.5 \mathrm{~V}$ bias, and it is evident that the first eigenchannels (Figure $7 b-e$ ) in the doped system have a substantial overlap in the surrounding of the $\mathrm{Si}$ and $\mathrm{S}$ atoms, which gives rise to the higher current in positive bias. This significant overlap might happen due to the delocalized charge density in the S-doped layer, which further gets pushed in the lower layer. The second eigenchannels now show much differences between pristine and doped nanojunctions. We see higher interaction in between the layers in dominant channels, and this somehow does not happen in armchair nanodevices. Eventually, we have a rectifying nanojunction in doped bilayer BP, and for making it into numbers, the overall rectification ratio is about 17 at $2.0 \mathrm{~V}$. This rectification can vary with the size of the nanoflake and the doping concentration.

This armchair nanojunction geometry, hence, shows a remarkable role played by the $\mathrm{Si}-\mathrm{S}$ couple on the transport properties. As any experimental nanojunction would also include possibly different doping structures, it would be interesting to see how the other relative arrangements of the $\mathrm{Si}$ and $S$ atom would influence the transport. We also focused on different arrangements; we changed the positions of paired doping in the device region. But the overall behavior was the same for the armchair direction, but in the zigzag direction, we obtained the lower rectification in most of the cases (details in the Supporting Information; Figures S2-S5). This lower rectification came from the weak coupling between the electrode and dopant state after changing the position. Further, if we change the position of dopants in such a way that they are far from each other, the overall rectifying behavior will disappear, unlike graphene. In the case of graphene, the charge density because of dopants is mostly delocalized throughout the layer, whereas in phosphorene, the charge densities are somehow more localized especially in the case of $S$ doping. Also, the interlayer interaction in phosphorene is higher than that of graphene; hence, the dopant state proves to be less dominating.

\section{CONCLUSIONS}

In conclusion, using DFT and NEGF calculations, we studied the paired substitutional doping in bilayer phosphorene. In different substitutional configurations, the Si substitution in the lower sublayer of the first layer and $S$ substitution in the upper sublayer of the second layer are the most favorable one. Contradictory to monolayer doping, bilayer doping exhibits a band gap smaller than that of pristine bilayer. Si and S dopants give the states on both sides of the Fermi, and it is not like standard p- and n-type doping. Further, the study of electronic transport properties of bilayer phosphorene along with doping shows the symmetric $I-V$ characteristics, yet resistivities are different along different directions. The overall current is high for the doped bilayer because the dopant starts taking part in the higher-bias regime, which further tunes the anisotropic behavior. The role played by the dopants on the interlayer current is discussed in nanojunction devices. The two symmetric nanojunctions oriented along armchair and zigzag directions with different resistivities are due to the anisotropy of the BP. We investigated the rectifying properties using eigenchannels and their localization across the nanojunction. No current rectification is observed in symmetric junctions, yet resistivities are different along different directions. Nanojunction devices exhibit lower current than the bilayer due to monolayer electrodes, but this reduction is lower than the previously discussed graphene nanojunction. It happens due to higher interlayer coupling in bilayer BP. $I-V$ characteristics for doped nanojunction shows symmetric behavior in the armchair direction but rectifying nature in the zigzag direction. We also studied two symmetric nanojunctions oriented along the armchair and zigzag directions with different resistivities due to the anisotropy of the BP. We further investigated the rectifying properties using eigenchannels and their localization across the junction. No current rectification is observed in symmetric junctions, yet resistivities are different along different directions. Further, the doped nanojunction in the armchair setup shows different current in opposite bias regimes, but this difference is really small and the change in current compared to pristine devices is not prominent enough. Higher bias transmission offers Fano resonance in armchair nanojunction, which comes from the hydrogen edge states coupling with the other layer. Interestingly, in the zigzag direction, the nanojunction device exhibits a good rectifying property with a rectification ratio $(I+/ I-)$ of 17 . This is because when an electron is getting transported from the Si-doped layer to the $\mathrm{S}$-doped layer, the current is higher than that of the other direction. The first eigenchannels clearly show that the doped system has a substantial overlap in $\mathrm{Si}$ and $\mathrm{S}$ atoms, which gives rise to higher currents in positive bias. Our study provides key insights into the electronic transport properties of bilayer BP nanojunctions and their usefulness for nanoelectronic applications.

\section{ASSOCIATED CONTENT}

\section{Supporting Information}

The Supporting Information is available free of charge at https://pubs.acs.org/doi/10.1021/acsaelm.0c00897.

Formation energies of dopants; calculated formation energy values (Table 1); top and side views of configurations of single and diatomic doping by $\mathrm{S}$ and $\mathrm{Si}$ in $\mathrm{Bi}-\mathrm{P}$ (Figure $\mathrm{S} 1$ ); bilayer armchair and zigzag phosphorene nanojunction device, zero-bias transmission function, and I-V characteristics (Figures S2-S5); and $I-V$ characteristics for varied doping positions (PDF)

\section{AUTHOR INFORMATION}

\section{Corresponding Author}

Vivekanand Shukla - Condensed Matter Theory Group, Materials Theory Division, Department of Physics and Astronomy, Uppsala University, Uppsala SE-75120, Sweden; (1) orcid.org/0000-0001-7724-6357;

Email: Vivekanand.Shukla@chalmers.se, vns391@ gmail.com

\section{Authors}

Rameshwar L. Kumawat - Department of Metallurgy Engineering and Materials Science, Indian Institute of 
Technology (IIT) Indore, Indore, Madhya Pradesh 453552, India; (1) orcid.org/0000-0002-2210-3428

Naresh K. Jena - Condensed Matter Theory Group, Materials Theory Division, Department of Physics and Astronomy, Uppsala University, Uppsala SE-75120, Sweden; (1) orcid.org/0000-0002-8242-8005

Biswarup Pathak - Department of Metallurgy Engineering and Materials Science and Department of Chemistry, Indian Institute of Technology (IIT) Indore, Indore, Madhya Pradesh 453552, India; 이이이.org/0000-0002-9972-9947

Rajeev Ahuja - Condensed Matter Theory Group, Materials Theory Division, Department of Physics and Astronomy, Uppsala University, Uppsala SE-75120, Sweden; Applied Materials Physics, Department of Materials Science and Engineering, Royal Institute of Technology (KTH), SE-10044 Stockholm, Sweden; 이이이.org/0000-0003-1231-9994

Complete contact information is available at:

https://pubs.acs.org/10.1021/acsaelm.0c00897

\section{Author Contributions}

V.S. and R.L.K. have contributed equally to this work.

Notes

The authors declare no competing financial interest.

\section{ACKNOWLEDGMENTS}

The authors (V.S., N.K.J., and R.A.) acknowledge computational resources provided through Swedish National Infrastructure for computing (SNIC2019-1-25), and R.L.K. acknowledges IIT Indore (India) for the lab and computing facilities. This work was supported by DST-SERB (Project number: CRG/2018/001131), CSIR [Grant number: 01(2886)/17/EMR(II)], and SPARC/2018-2019/P116/SL. V.S. acknowledges funding from the European Erasmus fellowship program (NAMASTE) and SSF grant (ITM170324), and R.L.K. acknowledges MHRD for senior research fellowship (SRF).

\section{REFERENCES}

(1) Geim, A. K.; Grigorieva, I. V. Van Der Waals Heterostructures. Nature 2013, 499, 419-425.

(2) Pumera, M.; Sofer, Z.; Ambrosi, A. Layered Transition Metal Dichalcogenides for Electrochemical Energy Generation and Storage. J. Mater. Chem. A 2014, 2, 8981-8987.

(3) Wang, Q. H.; Kalantar-Zadeh, K.; Kis, A.; Coleman, J. N.; Strano, M. S. Electronics and Optoelectronics of Two-Dimensional Transition Metal Dichalcogenides. Nat. Nanotechnol. 2012, 7, 699712.

(4) Novoselov, K. S.; Fal'ko, V. I.; Colombo, L.; Gellert, P. R.; Schwab, M. G.; Kim, K. A Roadmap for Graphene. Nature 2012, 490, 192-200.

(5) Rezapour, M. R.; Yun, J.; Lee, G.; Kim, K. S. Lower Electric Field-Driven Magnetic Phase Transition and Perfect Spin Filtering in Graphene Nanoribbons by Edge Functionalization. J. Phys. Chem. Lett. 2016, 7, 5049-5055.

(6) Rezapour, M. R.; Lee, G.; Kim, K. S. An Effective Approach to Realize Graphene Based p-n Junctions via Adsorption of Donor and Acceptor Molecules. Carbon 2019, 153, 525-530.

(7) Novoselov, K. S.; Geim, A. K.; Morozov, S. V.; Jiang, D.; Zhang, Y.; Dubonos, S. V.; Grigorieva, I. V.; Firsov, A. A. Electric Field Effect in Atomically Thin Carbon Films. Science 2004, 306, 666-669.

(8) Kubota, Y.; Watanabe, K.; Tsuda, O.; Taniguchi, T. Deep Ultraviolet Light-Emitting Hexagonal Boron Nitride Synthesized at Atmospheric Pressure. Science 2007, 317, 932-934.
(9) Mak, K. F.; Lee, C.; Hone, J.; Shan, J.; Heinz, T. F. Atomically Thin $\mathrm{MoS}_{2}$ : A New Direct-Gap Semiconductor. Phys. Rev. Lett. 2010, 105, No. 136805.

(10) Ramakrishna Matte, H. S. S.; Gomathi, A.; Manna, A. K.; Late, D. J.; Datta, R.; Pati, S. K.; Rao, C. N. R. $\mathrm{MoS}_{2}$ and $\mathrm{WS}_{2}$ Analogues of Graphene. Angew. Chem., Int. Ed. 2010, 49, 4059-4062.

(11) Zhang, J.; Xie, W.; Zhao, J.; Zhang, S. Band Alignment of TwoDimensional Lateral Heterostructures. 2D Mater. 2017, 4, No. 015038.

(12) Yu, H.; Kutana, A.; Yakobson, B. I. Carrier Delocalization in Two-Dimensional Coplanar $\mathrm{p}-\mathrm{n}$ Junctions of Graphene and Metal Dichalcogenides. Nano Lett. 2016, 16, 5032-5036.

(13) Khan, M. A.; Rathi, S.; Lee, C.; Kim, Y.; Kim, H.; Whang, D.; Yun, S. J.; Youn, D.-H.; Watanabe, K.; Taniguchi, T.; Kim, G.-H. High Performance Self-Gating Graphene/ $\mathrm{MoS}_{2}$ Diode Enabled by Asymmetric Contacts. Nanotechnology 2018, 29, No. 395201.

(14) Choi, M. S.; Qu, D.; Lee, D.; Liu, X.; Watanabe, K.; Taniguchi, T.; Yoo, W. J. Lateral $\mathrm{MoS}_{2} \mathrm{p}-\mathrm{n}$ Junction Formed by Chemical Doping for Use in High-Performance Optoelectronics. ACS Nano 2014, 8, 9332-9340.

(15) Chen, J.-W.; Lo, S.-T.; Ho, S.-C.; Wong, S.-S.; Vu, T.-H.-Y.; Zhang, X.-Q.; Liu, Y.-D.; Chiou, Y.-Y.; Chen, Y.-X.; Yang, J.-C.; Chen, Y.-C.; Chu, Y.-H.; Lee, Y.-H.; Chung, C.-J.; Chen, T.-M.; Chen, C.H.; Wu, C.-L. A Gate-Free Monolayer $\mathrm{WSe}_{2}$ p-n Diode. Nat. Commun. 2018, 9, No. 3143.

(16) Jin, C.; Kim, J.; Suh, J.; Shi, Z.; Chen, B.; Fan, X.; Kam, M.; Watanabe, K.; Taniguchi, T.; Tongay, S.; Zettl, A.; Wu, J.; Wang, F. Interlayer Electron-Phonon Coupling in $\mathrm{WSe}_{2} / \mathrm{hBN}$ Heterostructures. Nat. Phys. 2017, 13, 127-131.

(17) Lee, C.-H.; Lee, G.-H.; van der Zande, A. M.; Chen, W.; Li, Y.; Han, M.; Cui, X.; Arefe, G.; Nuckolls, C.; Heinz, T. F.; Guo, J.; Hone, J.; Kim, P. Atomically Thin $\mathrm{p}-\mathrm{n}$ Junctions with van Der Waals Heterointerfaces. Nat. Nanotechnol. 2014, 9, 676-681.

(18) Prasongkit, J.; Shukla, V.; Grigoriev, A.; Ahuja, R.; Amornkitbamrung, V. Ultrahigh-Sensitive Gas Sensors Based on Doped Phosphorene: A First-Principles Investigation. Appl. Surf. Sci. 2019, 497, No. 143660.

(19) Kumawat, R. L.; Garg, P.; Kumar, S.; Pathak, B. Electronic Transport through DNA Nucleotides in Atomically Thin Phosphorene Electrodes for Rapid DNA Sequencing. ACS Appl. Mater. Interfaces 2019, 11, 219-225.

(20) Kumawat, R. L.; Pathak, B. Individual Identification of DNA Nucleobases on Atomically Thin Black Phosphorene Nanoribbons: Van Der Waals Corrected Density Functional Theory Calculations. J. Phys. Chem. C 2019, 123, 22377-22383.

(21) Kumawat, R. L.; Pathak, B. Prospects of Black Phosphorus Nanoribbon for Explosive Sensing: A Computational Approach. Appl. Surf. Sci. 2020, 529, No. 147094

(22) Xiao, X.; Wang, M.; Tu, J.; Jiao, S. The Potential Application of Black and Blue Phosphorene as Cathode Materials in Rechargeable Aluminum Batteries: A First-Principles Study. Phys. Chem. Chem. Phys. 2019, 21, 7021-7028.

(23) Bian, S.; Wen, M.; Wang, J.; Yang, N.; Chu, P. K.; Yu, X.-F. Edge-Rich Black Phosphorus for Photocatalytic Nitrogen Fixation. J. Phys. Chem. Lett. 2020, 11, 1052-1058.

(24) Li, L.; Yu, Y.; Ye, G. J.; Ge, Q.; Ou, X.; Wu, H.; Feng, D.; Chen, X. H.; Zhang, Y. Black Phosphorus Field-Effect Transistors. Nat. Nanotechnol. 2014, 9, 372-377.

(25) Churchill, H. O. H.; Jarillo-Herrero, P. Phosphorus Joins the Family. Nat. Nanotechnol. 2014, 9, 330-331.

(26) Liu, H.; Neal, A. T.; Zhu, Z.; Luo, Z.; Xu, X.; Tománek, D.; Ye, P. D. Phosphorene: An Unexplored 2D Semiconductor with a High Hole Mobility. ACS Nano 2014, 8, 4033-4041.

(27) Shukla, V.; Grigoriev, A.; Ahuja, R. Rectifying Behavior in Twisted Bilayer Black Phosphorus Nanojunctions Mediated through Intrinsic Anisotropy. Nanoscale Adv. 2020, 2, 1493-1501.

(28) Sevik, C.; Wallbank, J. R.; Gulseren, O.; Peeters, F. M.; Cakır, D. Gate Induced Monolayer Behavior in Twisted Bilayer Black Phosphorus. 2D Mater. 2017, 4, No. 035025. 
(29) Cao, T.; Li, Z.; Qiu, D. Y.; Louie, S. G. Gate Switchable Transport and Optical Anisotropy in $90^{\circ}$ Twisted Bilayer Black Phosphorus. Nano Lett. 2016, 16, 5542-5546.

(30) Xie, J.; Luo, Q.; Jia, L.; Zhang, Z. Y.; Shi, H. G.; Yang, D. Z.; Si, M. S. Strain-Induced Recovery of Electronic Anisotropy in $90^{\circ}$ Twisted Bilayer Phosphorene. EPL 2018, 121, 27002.

(31) Ameen, T. A.; Ilatikhameneh, H.; Klimeck, G.; Rahman, R. Few-Layer Phosphorene: An Ideal 2D Material For Tunnel Transistors. Sci. Rep. 2016, 6, No. 28515.

(32) Rodin, A. S.; Carvalho, A.; Castro Neto, A. H. Strain-Induced Gap Modification in Black Phosphorus. Phys. Rev. Lett. 2014, 112, No. 176801.

(33) Fei, R.; Yang, L. Strain-Engineering the Anisotropic Electrical Conductance of Few-Layer Black Phosphorus. Nano Lett. 2014, 14, 2884-2889.

(34) Çakır, D.; Sahin, H.; Peeters, F. M. Tuning of the Electronic and Optical Properties of Single-Layer Black Phosphorus by Strain. Phys. Rev. B 2014, 90, No. 205421.

(35) Koenig, S. P.; Doganov, R. A.; Schmidt, H.; Castro Neto, A. H.; Özyilmaz, B. Electric Field Effect in Ultrathin Black Phosphorus. Appl. Phys. Lett. 2014, 104, No. 103106.

(36) Pierucci, D.; Henck, H.; Avila, J.; Balan, A.; Naylor, C. H.; Patriarche, G.; Dappe, Y. J.; Silly, M. G.; Sirotti, F.; Johnson, A. T. C.; Asensio, M. C.; Ouerghi, A. Band Alignment and Minigaps in Monolayer $\mathrm{MoS}_{2}$-Graphene van Der Waals Heterostructures. Nano Lett. 2016, 16, 4054-4061.

(37) Padilha, J. E.; Fazzio, A.; da Silva, A. J. R. Van Der Waals Heterostructure of Phosphorene and Graphene: Tuning the Schottky Barrier and Doping by Electrostatic Gating. Phys. Rev. Lett. 2015, 114, No. 066803.

(38) Guo, C.; Wang, T.; Xia, C.; Liu, Y. Modulation of Electronic Transport Properties in Armchair Phosphorene Nanoribbons by Doping and Edge Passivation. Sci. Rep. 2017, 7, No. 12799.

(39) Xie, F.; Fan, Z.-Q.; Zhang, X.-J.; Liu, J.-P.; Wang, H.-Y.; Liu, K.; $\mathrm{Yu}$, J.-H.; Long, M.-Q. Tuning of the Electronic and Transport Properties of Phosphorene Nanoribbons by Edge Types and Edge Defects. Org. Electron. 2017, 42, 21-27.

(40) Lv, Y.; Chang, S.; Huang, Q.; Wang, H.; He, J. Scaling Effect of Phosphorene Nanoribbon - Uncovering the Origin of Asymmetric Current Transport. Sci. Rep. 2016, 6, No. 38009.

(41) Shojaei, F.; Hahn, J. R.; Kang, H. S. Effect of Si-Si Bonds in Silicon-Doped $\alpha$-Phosphorene Bilayers: Two-Dimensional Layers and One-Dimensional Nanoribbons. J. Phys. Chem. C 2016, 120, 1710617114.

(42) Yu, W.; Zhu, Z.; Niu, C.-Y.; Li, C.; Cho, J.-H.; Jia, Y. Anomalous Doping Effect in Black Phosphorene Using FirstPrinciples Calculations. Phys. Chem. Chem. Phys. 2015, 17, 1635116358 .

(43) Guo, C.; Xia, C.; Fang, L.; Wang, T.; Liu, Y. Tuning Anisotropic Electronic Transport Properties of Phosphorene via Substitutional Doping. Phys. Chem. Chem. Phys. 2016, 18, 2586925878 .

(44) Lalitha, M.; Nataraj, Y.; Lakshmipathi, S. Calcium Decorated and Doped Phosphorene for Gas Adsorption. Appl. Surf. Sci. 2016, 377, 311-323.

(45) Boukhvalov, D. W. The Atomic and Electronic Structure of Nitrogen- and Boron-Doped Phosphorene. Phys. Chem. Chem. Phys. 2015, 17, 27210-27216.

(46) Lei, W.; Liu, G.; Zhang, J.; Liu, M. Black Phosphorus Nanostructures: Recent Advances in Hybridization, Doping and Functionalization. Chem. Soc. Rev. 2017, 46, 3492-3509.

(47) Wu, Z.-F.; Gao, P.-F.; Guo, L.; Kang, J.; Fang, D.-Q.; Zhang, Y.; Xia, M.-G.; Zhang, S.-L.; Wen, Y.-H. Robust Indirect Band Gap and Anisotropy of Optical Absorption in B-Doped Phosphorene. Phys. Chem. Chem. Phys. 2017, 19, 31796-31803.

(48) Pradhan, N. R.; Garcia, C.; Lucking, M. C.; Pakhira, S.; Martinez, J.; Rosenmann, D.; Divan, R.; Sumant, A. V.; Terrones, H.; Mendoza-Cortes, J. L.; McGill, S. A.; Zhigadlo, N. D.; Balicas, L.
Raman and Electrical Transport Properties of Few-Layered ArsenicDoped Black Phosphorus. Nanoscale 2019, 11, 18449-18463.

(49) Liu, B.; Köpf, M.; Abbas, A. N.; Wang, X.; Guo, Q.; Jia, Y.; Xia, F.; Weihrich, R.; Bachhuber, F.; Pielnhofer, F.; Wang, H.; Dhall, R.; Cronin, S. B.; Ge, M.; Fang, X.; Nilges, T.; Zhou, C. Black ArsenicPhosphorus: Layered Anisotropic Infrared Semiconductors with Highly Tunable Compositions and Properties. Adv. Mater. 2015, 27, 4423-4429.

(50) Osters, O.; Nilges, T.; Bachhuber, F.; Pielnhofer, F.; Weihrich, R.; Schöneich, M.; Schmidt, P. Synthesis and Identification of Metastable Compounds: Black Arsenic-Science or Fiction? Angew. Chem., Int. Ed. 2012, 51, 2994-2997.

(51) Long, M.; Gao, A.; Wang, P.; Xia, H.; Ott, C.; Pan, C.; Fu, Y.; Liu, E.; Chen, X.; Lu, W.; Nilges, T.; Xu, J.; Wang, X.; Hu, W.; Miao, F. Room Temperature High-Detectivity Mid-Infrared Photodetectors Based on Black Arsenic Phosphorus. Sci. Adv. 2017, 3, No. e1700589.

(52) Xu, Y.; Shi, Z.; Shi, X.; Zhang, K.; Zhang, H. Recent Progress in Black Phosphorus and Black-Phosphorus-Analogue Materials: Properties, Synthesis and Applications. Nanoscale 2019, 11, 14491-14527.

(53) Rezaee, A. E.; Kashi, M. A.; Baktash, A. Stone-Wales like Defects Formation, Stability and Reactivity in Black Phosphorene. Mater. Sci. Eng., B 2018, 236-237, 208-216.

(54) Giofré, D.; Ceresoli, D.; Fratesi, G.; Trioni, M. I. Electronic Transport in B-N Substituted Bilayer Graphene Nanojunctions. Phys. Rev. B 2016, 93, No. 205420.

(55) Hohenberg, P.; Kohn, W. Inhomogeneous Electron Gas. Phys. Rev. 1964, 136, B864-B871.

(56) Kohn, W.; Sham, L. J. Self-Consistent Equations Including Exchange and Correlation Effects. Phys. Rev. 1965, 140, A1133A1138.

(57) Kresse, G.; Joubert, D. From Ultrasoft Pseudopotentials to the Projector Augmented-Wave Method. Phys. Rev. B 1999, 59, 17581775.

(58) Perdew, J. P.; Burke, K.; Ernzerhof, M. Generalized Gradient Approximation Made Simple. Phys. Rev. Lett. 1996, 77, 3865-3868.

(59) Perdew, J. P.; Chevary, J. A.; Vosko, S. H.; Jackson, K. A.; Pederson, M. R.; Singh, D. J.; Fiolhais, C. Atoms, Molecules, Solids, and Surfaces: Applications of the Generalized Gradient Approximation for Exchange and Correlation. Phys. Rev. B 1992, 46, 66716687.

(60) Blöchl, P. E. Projector Augmented-Wave Method. Phys. Rev. B 1994, 50, 17953-17979.

(61) Berland, K.; Hyldgaard, P. Exchange Functional That Tests the Robustness of the Plasmon Description of the van Der Waals Density Functional. Phys. Rev. B 2014, 89, No. 035412.

(62) Soler, J. M.; Artacho, E.; Gale, J. D.; Garcia, A.; Junquera, J.; Ordejon, P.; Sanchez-Portal, D. The SIESTA Method for Ab Initio Order-N Materials Simulation. J. Phys.: Condens. Matter 2002, 14, 2745-2779.

(63) Artacho, E.; Anglada, E.; Diéguez, O.; Gale, J. D.; García, A.; Junquera, J.; Martin, R. M.; Ordejón, P.; Pruneda, J. M.; SánchezPortal, D.; Soler, J. M. The SIESTA Method; Developments and Applicability. J. Phys.: Condens. Matter 2008, 20, No. 064208.

(64) Troullier, N.; Martins, J. L. Efficient Pseudopotentials for Plane-Wave Calculations. II. Operators for Fast Iterative Diagonalization. Phys. Rev. B 1991, 43, 8861-8869.

(65) Monkhorst, H. J.; Pack, J. D. Special Points for Brillouin-Zone Integrations. Phys. Rev. B 1976, 13, 5188-5192.

(66) Brandbyge, M.; Mozos, J.-L.; Ordejón, P.; Taylor, J.; Stokbro, K. Density-Functional Method for Nonequilibrium Electron Transport. Phys. Rev. B 2002, 65, No. 165401.

(67) Datta, S. Electronic Transport in Mesoscopic Systems; Cambridge University Press, 1997.

(68) Çakır, D.; Sevik, C.; Peeters, F. M. Significant Effect of Stacking on the Electronic and Optical Properties of Few-Layer Black Phosphorus. Phys. Rev. B 2015, 92, No. 165406.

(69) Lei, S.; Wang, H.; Huang, L.; Sun, Y.-Y.; Zhang, S. Stacking Fault Enriching the Electronic and Transport Properties of Few-Layer 
Phosphorenes and Black Phosphorus. Nano Lett. 2016, 16, 1317-

1322.

(70) Frederiksen, T.; Paulsson, M.; Brandbyge, M.; Jauho, A.-P. Inelastic Transport Theory from First Principles: Methodology and

Application to Nanoscale Devices. Phys. Rev. B 2007, 75, No. 205413. 